\title{
Jean-Baptiste LE BOHEC, Norberto Bobbio et la question internationale
}

\section{Giovanni Carpinelli}

\section{(2) OpenEdition}

1 Journals

Édition électronique

URL : https://journals.openedition.org/ress/4149

DOI : $10.4000 /$ ress. 4149

ISSN : 1663-4446

Éditeur

Librairie Droz

\section{Édition imprimée}

Date de publication : 14 décembre 2018

Pagination : 286-288

ISSN : 0048-8046

\section{Référence électronique}

Giovanni Carpinelli, « Jean-Baptiste LE BOHEC, Norberto Bobbio et la question internationale », Revue européenne des sciences sociales [En ligne], 56-2 | 2018, mis en ligne le 14 décembre 2018, consulté le 07 janvier 2022. URL : http://journals.openedition.org/ress/4149; DOI : https://doi.org/10.4000/ress. 4149

Ce document a été généré automatiquement le 7 janvier 2022.

(c) Librairie Droz 


\title{
Jean-Baptiste LE BOHEC, Norberto Bobbio et la question internationale
}

\author{
Giovanni Carpinelli
}

\section{RÉFÉRENCE}

Jean-Baptiste LE BOHEC, 2016, Norberto Bobbio et la question internationale, Rennes,

Presses Universitaire de Rennes, 402 p.

1 La réputation du philosophe turinois Norberto Bobbio (1909-2004) ne cesse de grandir dans le monde. Son nom est normalement associé à la théorie du droit et à une reflexion sur la démocratie. Il fut aussi un auteur Droz (On Mosca and Pareto, 1972) et collabora à la Revue européenne des sciences sociales (par quatre articles, de 1979 à 1981). Moins connus sont ses travaux, la plupart occasionnels, sur les relations internationales, dont deux furent traduits en français dans son ouvrage de 1999 L'État et la démocratie internationale (Bruxelles, Complexe). Jean-Baptiste Le Bohec s'appuie sur l'ensemble de ces textes et en délivre une analyse systématique qui dépasse assurément les intentions de Bobbio lui-même - ce dernier n'ayant jamais visé à faire une théorie méthodique des relations internationales (voir Mario G. Losano, Norberto Bobbio. Una biografia culturale, Rome, Carocci, 2018, p. 195) -, mais dont on ne saurait sous-estimer l'intérêt.

2 Issu d'une thèse de doctorat soutenue à l'université de Rennes 1 en mai 2013, Norberto Bobbio et la question internationale se compose de cinq parties. La première ( Critique du marxisme ») est centrée sur les questions que Bobbio pose au marxisme ou, pour mieux dire, à certains marxistes depuis son livre, sans doute le plus fameux, Politica e cultura de 1955. À bien d'égards, soutient-il, le marxisme n'a pas tenu ses promesses : cela est vrai, entre autres choses, de sa prévision d'un dépérissement progressif de l'État, mais ça l'est aussi de sa contribution à l'étude des relations internationales. La théorie léniniste de l'impérialisme en fournit la preuve tant il est vrai que les guerres ont rarement des causes économiques. Selon Bobbio, elles relèvent principalement de la 
politique de puissance des États, même des plus petits, dès qu'ils en ont l'opportunité. Cela confère à l'ordre international le caractère d'un désordre permanent, d'une anarchie.

3 C'est ce qu'expose la deuxième partie de l'ouvrage («Philosophie des relations internationales ») axée sur le long article de 1966 «Il problema della guerra e le vie della pace» (repris en 1979 dans un recueil du même titre) et qui consacre un chapitre au rapport ami-ennemi à partir de la correspondance entretenue par Bobbio avec Carl Schmitt de 1948 à 1980 (publiée par Piet Tommissen en 1995 dans la revue Diritto e Cultura). L'article de 1966 défend le principe d'un pacifisme actif - détaillé selon ses formes instrumentale, institutionnelle et finaliste - comme moyen pour atteindre la paix. La voie du pacifisme actif est praticable à deux conditions. Cette deuxième partie présente la première de ces conditions. Elle consiste en un changement des régimes des États qui ignorent ou déprécient le pluralisme des partis et la séparation des pouvoirs. Les États sont tous appelés à se doter d'institutions démocratiques et représentatives : «bien que l'anarchie internationale serve de point de départ à la théorie bobbienne des relations internationales, cette situation n'est pas indépassable. À la différence des réalistes, qui jugent ce système incurablement anomique, il s'agit pour le philosophe d'y faire progresser le droit et la démocratie. » (p. 137).

4 La troisième partie («Philosophie de l'histoire») montre que l'objectif d'une paix durable tire sa solidité, chez Bobbio, du fait qu'il va de pair avec une philosophie de l'histoire explicite qui, comme toutes les philosophies du même genre, comporte une idée de progrès que Bobbio, suivant en cela l'exemple de Kant, comprend justement comme extension du droit. Les philosophies de l'histoire s'attachent traditionnellement à expliquer la constance du mal, à lui donner un sens et donc à légitimer la guerre qui est la plus universelle des relations funestes entre les peuples. La condition atomique change pourtant la donne d'après Bobbio. En effet, «comment justifier encore une guerre à laquelle rien ne peut succéder ?» (p. 167). «La force que les philosophies de l'histoire exercent, et l'impasse à laquelle elles conduisent l'humanité [...] sont liées selon Bobbio à leur formidable travail de justification de la guerre, qui s'enracine profondément dans notre psychisme collectif. Asservis à ces croyances, nous adhérons malgré nous à ces justifications.» (p.179). Bobbio invite à tourner le dos à ces philosophies et à aller plus loin. Un monde en danger requiert une vision renouvelée des fins vers lesquelles tendrait l'histoire et il va de soi que ces fins découlent de valeurs indémontrables par définition. On peut néanmoins soutenir rationnellement le besoin de recourir à ces valeurs (p. 147). La quatrième partie («Les fins de l'histoire ») expose alors comment Bobbio conçoit l'histoire et le dessein auquel elle obéirait : elle est à la fois histoire de la liberté et histoire de l'égalité. Dans cette perspective, la diffusion atteinte par les droits de l'homme reflète le niveau du progrès social.

Reste à établir quelle est la seconde condition à laquelle la voie du pacifisme actif doit se soumettre pour être praticable, en plus de la démocratisation des régimes autoritaires. C'est l'objet de la cinquième et dernière partie de l'ouvrage de Le Bohec («Le droit et la démocratie internationale») qui précise qu'elle réside dans l'organisation fédérale de la collectivité internationale, « une société de sociétés » pour le dire avec le Montesquieu du Livre IX de L'Esprit des lois : les États, tout en demeurant indépendants, transfèrent des compétences à des institutions communes. Bobbio renoue ici avec les pères du fédéralisme qui lui sont chers, dont son Carlo Cattaneo. Un chapitre de cette partie traite du «Tiers » en politique, d'après un livre de Bobbio sur 
ce même sujet (Il Terzo assente, 1989) et défend l'idée que dans l'attente d'une pleine transformation fédéraliste du système international, la création d'un pouvoir tiers audessus des parties serait en mesure de résoudre les conflits entre les États, ou du moins de s'y essayer. Ce Tiers existe déjà : ce sont les Nations unies. Sa relative « absence » en souligne l'inefficacité trop souvent constatée.

6 On s'en tiendra pour conclure à ces quelques remarques de forme. D'assez nombreuses coquilles émaillent le texte, sans pour autant nuire à son intelligibilité, sauf peut-être dans deux cas : «celer " pour "sceller » (p. 208) ou «emprunt » pour «empreint " (p. 334). Des imprécisions sont également à signaler au début de l'ouvrage. La grève d'août 1922 ne fut pas « la dernière réaction populaire majeure contre la politique des fascistes au pouvoir » (p.12) étant donné que la "marche sur Rome», qui ouvre au fascisme les portes du pouvoir, date du mois d'octobre de la même année. Bobbio ne suivit pas les cours de Benedetto Croce, Piero Gobetti et Piero Calamandrei à l'université de Turin à partir de 1927 (p. 12). Et pour cause : Croce et Gobetti (ce dernier mort, qui plus est, en 1926) n'étaient pas des universitaires, tandis que Calamandrei, quant à lui, enseignait à l'université de Florence.

\section{AUTEURS}

\section{GIOVANNI CARPINELLI}

Turin, Fondazione Istituto piemontese Antonio Gramsci 